\title{
Decoding the role of chromatin architecture in development: coming closer to the end of the tunnel
}

\author{
Chongyuan Luo ${ }^{1+\neq}$, Juan Dong ${ }^{1,2 * \neq}$, Yi Zhang ${ }^{1 \dagger}$ and Eric Lam ${ }^{1 *}$ \\ ${ }^{1}$ Department of Plant Biology and Pathology, Rutgers the State University of New Jersey, New Brunswick, NJ, USA \\ ${ }^{2}$ The Waksman Institute of Microbiology, Rutgers the State University of New Jersey, Piscataway, NJ, USA
}

\section{Edited by:}

Olga Pontes, University of New

Mexico, USA

\section{Reviewed by:}

Crisanto Gutierrez, Consejo Superior de Investigaciones Cientificas, Spain Yuehui He, National University of

Singapore, Singapore

\section{${ }^{*}$ Correspondence:}

Eric Lam, Department of Plant Biology and Pathology, Rutgers the State University of New Jersey, 59 Dudley Road, New Brunswick, NJ 08901, USA

e-mail: lam@aesop.rutgers.edu; Juan Dong, The Waksman Institute of Microbiology, Rutgers the State University of New Jersey, Piscataway, NJ 08854, USA

e-mail:dong@waksman.rutgers.edu

\section{${ }^{\dagger}$ Present address:}

Chongyuan Luo, Plant Biology Laboratory, The Salk Institute for

Biological Studies, La Jolla, CA 92037, USA;

Yi Zhang, Section of Cell and

Developmental Biology, University of

California San Diego, La Jolla,

CA 92093, USA

${ }^{\ddagger}$ Chongyuan Luo and Juan Dong have contributed equally to this work.
Form and function in biology are intimately related aspects that are often difficult to untangle. While the structural aspects of chromatin organization were apparent from early cytological observations long before the molecular details of chromatin functions were deciphered, the extent to which genome architecture may impact its output remains unclear. A major roadblock to resolve this issue is the divergent scales, both temporal and spatial, of the experimental approaches for examining these facets of chromatin biology. Recent advances in high-throughput sequencing and informatics to model and monitor genome-wide chromatin contact sites provide the much-needed platform to close this gap. This mini-review will focus on discussing recent efforts applying new technologies to elucidate the roles of genome architecture in coordinating global gene expression output. Our discussion will emphasize the potential roles of differential genome 3-D structure as a driver for cell fate specification of multicellular organisms. An integrated approach that combines multiple new methodologies may finally have the necessary temporal and spatial resolution to provide clarity on the roles of chromatin architecture during development.

Keywords: chromatin, chromatin beacons, chromatin conformation capture, INTACT method, cell fate specification

\section{INTRODUCTION}

Chromatin is the de facto genetic material of eukaryotic cells, which is composed of an extraordinarily complex array of nucleoprotein components. Numerous studies in the past decades have suggested that the transcription activity across the genome can be correlated with a number of microscopic features, such as chromatin compactness (Beisel and Paro, 2011), proximity of the locus to the nuclear laminar, nuclear pore or nucleolus (Misteli, 2007; Berman et al., 2011), and the mobility of chromatin (Chuang et al., 2006; Rosin et al., 2008). However, it has been difficult to unambiguously integrate microscopic data of chromatin behavior with the mechanisms of transcription regulation and epigenetic control. The recent advent of sequencing-based genome-wide chromosome conformation capture techniques (such as $3 \mathrm{C}$ or $\mathrm{Hi}-\mathrm{C}$ ) provided the much-needed tools to relate long-range chromatin interactions with other genomic and epigenomic features (Dekker et al., 2002; Lieberman-Aiden et al., 2009). However, the structural basis of $3 \mathrm{C}$ and $\mathrm{Hi}-\mathrm{C}$ is not completely understood. In particular, it remains unresolved whether the chromatin interactions detected by $3 \mathrm{C}$ or $\mathrm{Hi}-\mathrm{C}$ reflected molecular $(10-100 \mathrm{~nm})$ or cytological (100-1000 nm) proximity (Belmont, 2014).

Complement to the ligation-based proximity assays, tagging of genomic loci with chromatin beacons provides the means of measuring chromatin dynamics and spatial relevance to ultrastructures (Heun et al., 2001; Kato and Lam, 2001; Lam etal., 2004; Rosin et al., 2008). For studying chromatin conformation in polyploid plant species or endoreduplicated plant cells, this approach can enable the tracking of highly homologous alleles as shown in studies using mapped transgenic Arabidopsis thaliana plants (Rosin et al., 2008; Rosa et al., 2013). However, the introduction of chromatin beacons may associate with potential caveats, such as the large transgene construct can be targeted by gene silencing machinery in some cases and therefore alter the local chromatin context (Watanabe et al., 2005; Jovtchev et al., 2008, 2011).

In this mini-review, we will first summarize the global features revealed by Hi-C assays, followed by describing possible biological roles of chromatin architecture in cellular differentiation 

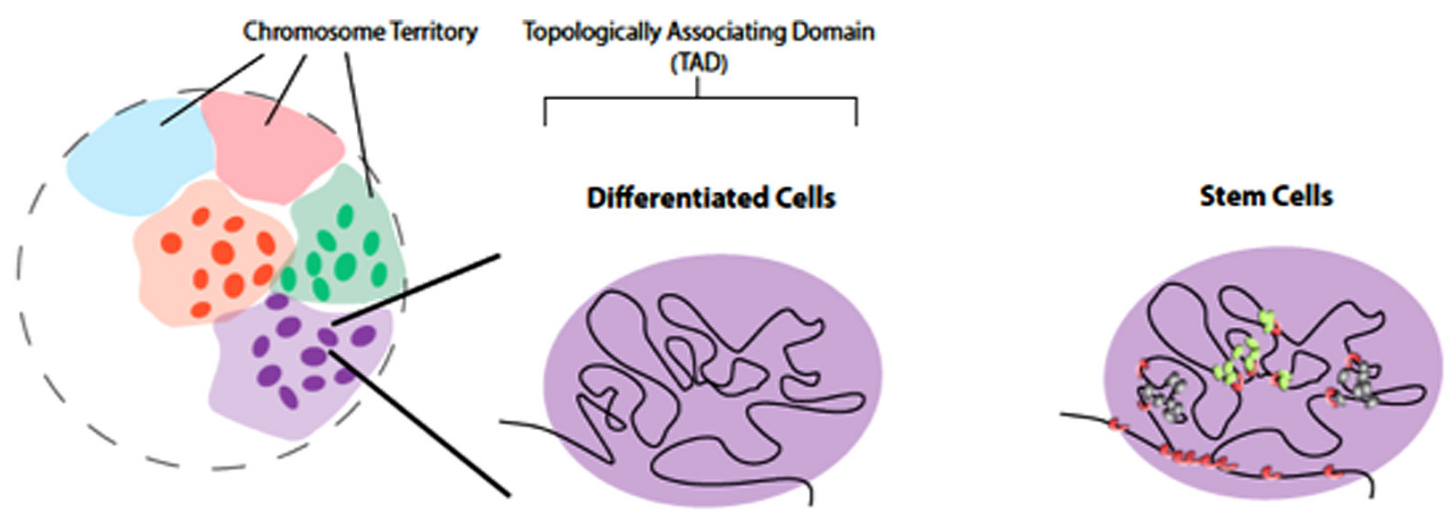

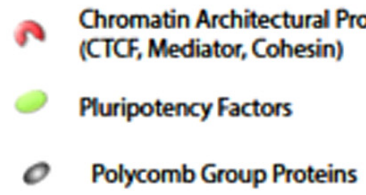

Chromatin Architectural Proteins (CTCF, Mediator, Cohesin)

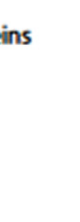

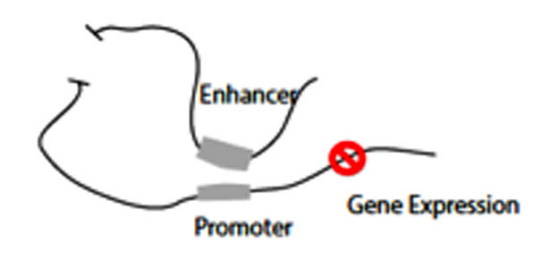

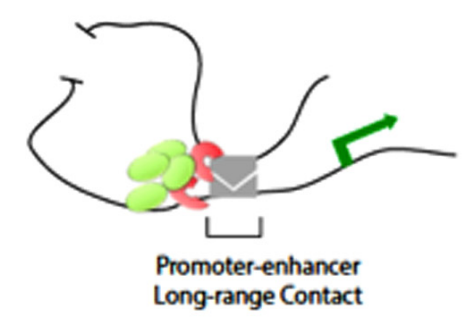

FIGURE 1 | Chromatin organization and topological domains. Chromosomes occupy distinct territories in the nucleus (shaded with different colors). Long-range chromatin looping, at the sub-megabase level, partitions the chromosomal region into Topologically Associating Domains (TADs; individuals illustrated on the rights. The TADs remain largely unchanged in differentiated cells and stem cells. However, at a finer levH pluripotency factors and chromatin architectural proteins organize higher-order chromatin connectivity during reprogramming. Pluripotency factors co-localize and occupy distinct spatial regions from PcG proteins in stem cells. Such chromatin reorganization induced by pluripotency factors is important for cell-specific gene expression. and during development. We will focus on a number of genetic studies involving genes encoding chromatin modifiers and conclude with perspectives of deploying cell-type-specific techniques to better resolve the functional consequence of the chromatin interactome.

\section{GLOBAL PATTERNS OF CHROMATIN ORGANIZATION}

Recent Hi-C experiments performed with animal cells (Lieberman-Aiden et al., 2009; Dixon et al., 2012; Sexton et al., 2012) have confirmed the existence of chromosome territories that were previously described cytologically (Figure 1). The probabilities of intra-chromosomal interaction, even for loci that are $200 \mathrm{Mb}$ apart, are much greater than that of interchromosomal interactions. This characteristic of Hi-C data has been utilized to assist genome-wide haplotype reconstruction and genome assembly scaffolding (Burton et al., 2013; Kaplan and Dekker, 2013; Selvaraj et al., 2013). In both insects and mammals that have been studied, the vast majority of the genome is organized into large topological domains with median size of $\sim 100 \mathrm{~kb}$ for Drosophila or $\sim 1 \mathrm{Mb}$ for human. Considering the $20 \times$ difference between the Drosophila and human genome sizes, the scale of topological domains are largely comparable (Dixon et al., 2012; Sexton et al., 2012). Several types of genome compartmentalization mechanisms may be involved to create and maintain topological domains. For example, the binding of CTCF (CCCTC-binding factor, insulator binding protein) and a repressive histone mark $\mathrm{H} 3 \mathrm{~K} 9 \mathrm{me} 3$ were found enriched at the boundary of topological domains (Dixon et al., 2012). Large scale genome features such as lamina-associated domains and latereplicating-chromatin were also found to partially overlap with topological domain boundaries, suggesting that nuclear ultrastructure and cell cycle control may contribute to the formation of such domains.

A striking finding from studies with both Drosophila and mammals was that topological domains appear to be stable across cell types and were also conserved between mouse and human (Dixon etal., 2012; Sexton etal., 2012). This finding suggests that the mechanism maintaining genome topological domains is likely ancient in the animal kingdom. With greater sequencing depth $(150 \times$ coverage of the haploid genome) and improved data processing algorithms, chromatin interactions could be determined with a resolution of 5-10 kb for the human genome (Jin et al., 2013). Unlike the stable long-range topological domains, the observed short-range interactions are highly cell-type specific, presumably reflecting specific regulatory activities such as enhancer-promoter interactions. An intriguing finding was that perturbation of the cellular state with a signaling molecule did not significantly alter genomic patterns of short-range looping (Jin etal., 2013), which may indicate that enhancer-promoter interaction is integral to programming cellular identity and may be primed before the signaling event to facilitate timely cellular response. 
In plants, global chromatin organization of Arabidopsis thaliana has been studied with $4 \mathrm{C}$ method using 13 viewpoints covering each chromosome and context (Grob et al., 2013). With this 4C dataset, the work showed that intra-arm interactions are $10 \times$ more abundant than inter-arm interactions, which are also about $10 \times$ more frequent than inter-chromosomal interactions. Consistent with the findings in human, inter-arm and inter-chromosomal interactions are more likely to occur between regions that are distant to the centromere, which are also gene rich and actively expressed. However, one exception to this trend is that strong interactions were observed between the heterochromatin knob on chromosome 4 of Arabidopsis and pericentromeres on various chromosomes in addition to that on chromosome 4. This observation suggests that epigenetic mechanisms such as histone modifications and cytosine methylation may also modulate large-scale interactions in the genome. Since plant species are known to adopt different interphase chromosome configurations that may be correlated with genome sizes and/or ploidy levels (Dong and Jiang, 1998; Cowan etal., 2001; Santos and Shaw, 2004), whether the organization pattern observed by Grob et al. (2013) can be generalized to other plant genomes remains to be determined.

Using time-lapse fluorescent imaging of four chromatin beacon lines, each containing a single copy of an identical transgene that has been mapped to a $100 \mathrm{~kb}$ region adjacent to the nucleolus organizing region (NOR) on Arabidopsis chromosome 2, we obtained evidence consistent with a topological domain (Rosin et al., 2008). This proposed domain shares several characteristics with topological domains described in insects and mammals: (1) The size of the domain we observed is similar to the average chromatin domain found in Drosophila, which is consistent with the comparable genome size of Arabidopsis and Drosophila. (2) The chromatin beacon line residing at the domain boundary (CCT432) appeared much closer to the NOR in 3-D compared to the other three beacons in the proposed domain. The result suggests that association with the nucleolus helps to create and/or maintain the topological domain. This model is akin to observed cases in which topological domain boundaries overlap with laminaassociated domains. (3) The reporter gene in line CCT432, as well as the flanking genes surrounding the transgene in this line, is actively expressed. This is consistent with the observation that housekeeping genes are enriched in topological domain boundaries.

\section{THE REGULATORY FUNCTION OF CHROMATIN ORGANIZATION}

In animal systems, enhancers are distal regulatory elements that can locate from several $\mathrm{kb}$ to over one $\mathrm{Mb}$ away from the promoter that is being modulated. Enhancers are typically marked by $\mathrm{H} 3 \mathrm{~K} 4 \mathrm{mel}$ and $\mathrm{H} 3 \mathrm{~K} 27 \mathrm{Ac}$ and are depleted of H3K4me3 (Creyghton et al., 2010). Subsets of enhancers are also bound by the co-activator CBP/P300 and/or mediator components (Visel et al., 2009). Enhancers can regulate distant promoters through creating spatial proximity by looping, with involvement of the Cohesin machinery (Kagey et al., 2010). Studies with RNA polymerase II centered ChIA-PET and Hi-C experiments have revealed that distinct cell types can display different subsets of enhancer-promoter interactions (Zhang et al., 2012; KiefferKwon et al., 2013). Mirroring the finding from Hi-C assays that short-range looping is not affected by cellular stimulations (Jin et al., 2013), activating the TNF- $\alpha$ pathway in human fetal lung fibroblast cells (IMR90) does not cause significant remodeling of enhancer-promoter contacts. Therefore, cell-type-specific signaling programs may be established by chromatin architectural features found in particular cell types.

In contrast to the case in mammalian systems, evidence supporting gene regulation by distal elements as a prominent mechanism in plants is scarce. Enhancer traps have been successfully applied in plant systems for the establishment of cell-type-specific marker lines (Haseloff, 1999). However, the responsible regulatory sequences are rarely cloned and analyzed with respect to distant regulatory element - promoter pairs.

A number of recent studies in plants using both proximity ligation and chromatin beacon strategies have suggested chromatin organizations may regulate gene functions for specific pathways. The characterization of regulatory elements at the maize booster (b1) locus provided the most convincing example of a plant distal regulatory element to date. Among many alleles of the b1 locus, B-I and $\mathrm{B}^{\prime}$ were found to contain identical DNA sequences but are associated with distinct chromatin and expression characteristics and were therefore considered epi-alleles (Louwers et al., 2009). The repressed B' allele was further found to be paramutagenic, which is capable of converting the actively expressed $\mathrm{B}-\mathrm{I}$ to an inactive $\mathrm{B}^{\prime}$ allele when combined genetically. A hepta-repeat locating $\sim 100 \mathrm{~kb}$ upstream of the b1 transcription start site (TSS) fits the classical definitions of a distal enhancer element: (1) The presence of multiple $853 \mathrm{bp}$ repeats was required for activated b1 transcription. (2) Using the 3C assay, the hepta-repeats were found to physically interact with the b1 TSS specifically in tissues where b1 is actively expressed. In addition, the authors also found two more restriction fragments each located $\sim 47 \mathrm{~kb}$ and $107 \mathrm{~kb}$ upstream that can physically interact with the b1 TSS (Louwers et al., 2009). These two regions interact with the b1 TSS in a manner that correlates with b1 expression levels, suggesting that they may function as novel distal regulatory elements.

Two recent studies analyzed the chromatin conformation of the Arabidopsis FLC locus using the complementary 3C and chromatin-beacon approaches. A physical gene loop was discovered between the $5^{\prime}$ - end of FLC and a region several 100 base pairs downstream of the FLC polyadenylation site (Crevillén et al., 2013). Intriguingly, the gene loop is disrupted at an early stage of vernalization ( 2 weeks in the cold) and remains disrupted after returning to warm temperatures for 7 days. This "cold memory" behavior of the observed loop suggests it can either be a carrier of epigenetic information or it is responsive to other epigenetic inputs. Using a chromatin beacon strategy, Rosa et al. (2013) investigated the physical interactions of multiple FLC alleles in endoreduplicated Arabidopsis root cells. Physical clustering of $F L C$ alleles was observed in response to vernalization. Similarly to disruption of the $F L C$ gene loop, clustering of $F L C$ alleles also remains stable at least 7 days after plants were returned to warmer conditions. 


\section{3-D CHROMATIN ARCHITECTURE AND STEM CELL PLURIPOTENCY}

Stem cells are uniquely endowed with the capability of infinite self-renewal and a wide differentiation potential to generate multiple cell types. Plants and animals both rely on stem cell populations for embryogenesis and post-embryonic development. In Arabidopsis, the major stem cell populations are maintained in the shoot and root apical meristems (SAM and RAM), respectively. Extrinsic cues (phytohormones, wounding, etc.) and signals from stem cell niches have been shown to modulate stem cell activities and to shape plant development (Sablowski, 2004; Singh and Bhalla, 2006). In terms of intrinsic pluripotency factors in plants, the homeodomain transcription factors WUSCHEL (WUS) and SHOOT MERISTEMLESS (STM), when ectopically expressed, induce cellular reprogramming and ectopic shoot stem cell proliferation (Gallois et al., 2002). In RAM, stem cell population is defined by the longitudinal gradient of PLETHORA (PLT) 1 and 2, as well as the APETALA 2 (AP2) transcription factors, and the radial gradient of the SHORTROOT (SHR) and SCARECROW (SCR) transcription factors (Sabatini etal., 2003; Aida etal., 2004; Galinha etal., 2007). Expression of both PLT and SCR can induce ectopic formation of root stem cells (Aida et al., 2004; Galinha et al., 2007).

Extensive studies have shown how pluripotency transcription factors function together and are regulated by chromatin regulators to control cell identity (Papp and Plath, 2013). It has been hypothesized that transcription regulatory circuits and epigenetic modification patterns are involved in pluripotency induction and stem cell maintenance (Lessard and Crabtree, 2010; Mattout and Meshorer, 2010; Young, 2011). Stem cells can also display distinct features in DNA methylation (Meissner, 2010), histone modification, chromatin remodeling (Chen and Dent, 2014; Thiagarajan et al., 2014), and transcription regulation via non-coding RNA-based mechanisms (Wright and Ciosk, 2013; Bergmann and Spector, 2014). Compared to genomes of differentiated cells, those of stem cells in general have more open chromatin configuration and dynamic association with chromatin proteins, perhaps reflecting their plasticity in self-renewal and pluripotency (Meshorer et al., 2006; Mattout and Meshorer, 2010). The stem cell genomes of mammals are also featured with bivalent chromatin configuration, the co-enrichment of functionally opposite chromatin marks (repressive H3K27me3 and activating H3K4me3) at the TSS of many transcription factors and signaling components (Vastenhouw et al., 2010; Voigt et al., 2013). This promoter "poising" may facilitate more rapid cell fate commitment upon reception of developmental cues by the stem cell populations.

Several recent studies suggested that "form-precedingfunction," where 3-D reorganization of chromatin may occur before detectable transcriptional and phenotypic changes during pluripotency induction. The formation of 3-D chromatin loops by long-range promoter-enhancer associations may coordinate cell type-specific gene expression (Deng et al., 2012; Li et al., 2012; Zhang et al., 2013). In addition, pluripotency factors (Oct4, Nanog, and Sox2) may help to reorganize higher-order chromatin connectivity (Apostolou et al., 2013; de Wit et al., 2013;
Ginno and Schubeler, 2013; Krijger and de Laat, 2013) through colocalization and creating long-range contacts in pluripotent stem cells (Figure 1). Chromatin architectural proteins (CTCF, Mediator, and Cohesin), in a combinatorial manner, may facilitate the pluripotency factors to bridge cell type-specific chromatin looping (Phillips-Cremins et al., 2013). Indeed, while the overall topologically associating domains (TADs) largely remain unchanged between stem cells and differentiated cells (Dixon et al., 2012; Nora et al., 2012), the genome of pluripotent stem cells displays unique 3-D contacts that are dependent on the expression of pluripotency factors and the chromatin insulators (Apostolou et al., 2013; de Wit et al., 2013; Wei et al., 2013). In addition, recent studies showed that another layer of regulation provided by the PcG (polycomb group) proteins is working to regulate chromatin 3-D organization in mouse stem cells during reprogramming (Denholtz et al., 2013).

\section{CELL FATE RESETTING IN PLANTS}

In Arabidopsis, stem cell transcription factors, like pluripotency factors in mammals, are sufficient to induce shoot or root stem cells ectopically. It is tantalizing to speculate that they may also be involved in architectural reorganization of chromatin in the process of cell fate determination in plants. As the topological domains in Arabidopsis await to be further established with more

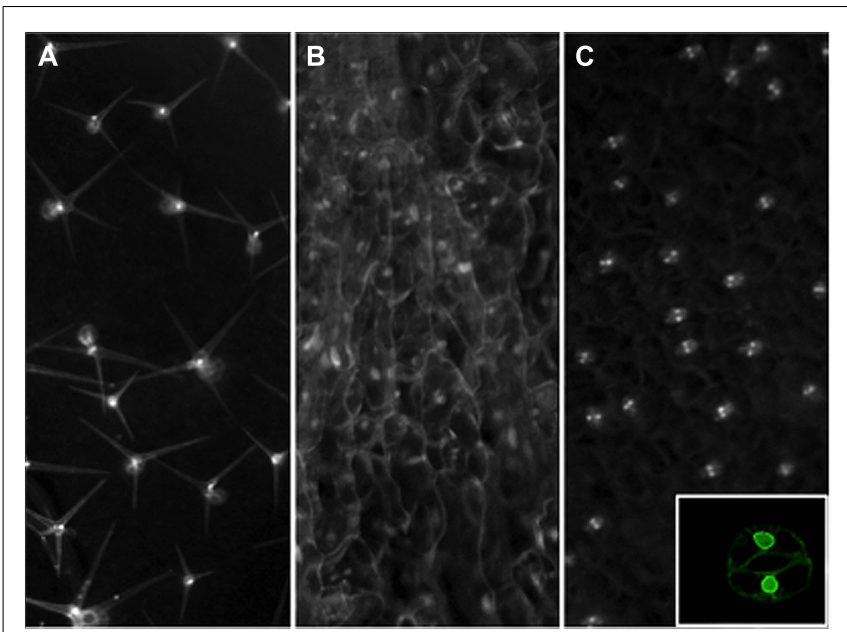

FIGURE 2 | Nuclear envelope tagging of specific leaf cell types in Arabidopsis. Transgenic plants were created with Inserts that express an NTF marker gene driven by the GL2 (A), CaMV35S (B), or AtMYB60 (C) promoters for trichome, constitutive, or guard cell-specific expression. The NTF marker is a nuclear envelope-anchored GFP fused to a biotinylation epitope from bacteria. For the GL2 promoter driven construct, a separate vector is used to produce the bacterial BirA gene in trans to catalyze the biotinylation of the NTF in order to facilitate rapid nuclei purification (Deal and Henikoff, 2010). To create a more facile labeling system, we have generated a new INTACT vector that contains both the NTF expression cassette as well as the BirA gene for plant expression. The CaMV 35S promoter and a guard cell-specific promoter from the AtMYB60 gene are used to create the constructs shown in panels (B) and (C), respectively. Mature rosette leaves from stable transgenic lines are examined by a stereo epifluorescence microscope fitted with a GFP filter set for images shown. Inset on panel (C) shows a confocal image of a tagged guard cell from a single focal plane to Illustrate the nuclear envelope localization of the expressed NTF protein from the new vector driven by the AtMYB60 promoter. 
3C and Hi-C studies, here we will discuss recent evidence for linking plant pluripotency factors to epigenetic regulation of stem cell maintenance. Several review articles summarized a wide spectrum of chromatin modifiers and remodelers and their roles in the maintenance of stem cell fate in plants (Kornet and Scheres, 2008; Sang et al., 2009; Shen and Xu, 2009). For example, a histone acetyltransferase AtGCN5 has been shown to regulate both shoot and root stem cells via controlling the expression of WUS and PLT, respectively. Another conserved chromatin remodeler, the SWI/SNF-type ATPase factor, SPLAYED (SYD), was shown to directly bind to the WUS promoter for activation of WUS expression in the SAM (Kwon et al., 2005). The shoot pluripotency factor STM is suppressed by CURLY LEAF (CLF), a PcG protein, and deposition of H3K27me3 marks at the promoter region (Katz et al., 2004; Schubert et al., 2006). A recent study demonstrated that CLF also suppresses stem cell resetting in stomatal development (Lee et al., 2014). Mature guard cells (GCs), a terminal cell type in the epidermis, are once thought to be irreversibly differentiated. A recent study showed that cell fate of mature GCs can be reset by expression of two transcription factors, FAMA and FOUR LIPS (FLP), resulting in a stoma-in-stoma phenotype. Constitutive expression of CLF abrogated this cell fate resetting, apparently by silencing the stomatal stem cell genes SPEECHLESS (SPCH) and MUTE, which encode bHLH transcription factors (Lee et al., 2014). These genetic studies in plant development indicate the potential involvement of chromatin modifiers/regulators in early steps of cell fate specification. Whether genome architecture per se is a driver for this developmental commitment is a key question that needs to be resolved.

\section{FUTURE DIRECTIONS}

While the 3C-derived chromatin proximity assay and chromatin beacon platform in Arabidopsis open up opportunities to close the physical scale gap for studies on chromatin architecture and function, the coexistence of multiple cell types is a confounding issue that often limits the interpretations that can be derived. A facile method for rapid purification of intact nuclei from specific cell types at different stages will greatly augment these techniques by providing additional specificities. The INTACT (isolation of nuclei tagged in specific cell types) method was demonstrated to enable production of cell-type-specific ChIP-seq in Arabidopsis root cells (Deal and Henikoff, 2010), as well as nucleosome occupancy maps for muscle cells of mature Caenorhabditis elegans and mesoderm cells of Drosophila embryos (Steiner et al., 2012). As shown in Figure 2, this method can be readily adapted to specifically tag distinct populations of epidermis-derived leaf cell types such as trichomes and GCs in Arabidopsis. Using this approach, we can now deploy development time- and cell type-specific promoters to enable the rapid isolation of nuclei from distinct cell types at defined steps of development. The integration of this method with other genome-wide, sequence-based techniques as well as the chromatin beacon resource should narrow the temporal gap in our methods to study the architecture of chromatin at a defined developmental time and space. We are hopeful that this fusion of new methodologies should finally bring us closer to mapping the early changes in chromatin organization that may drive predictable genome outputs and cell fates.

\section{ACKNOWLEDGMENTS}

The work on chromatin in the Lam lab was supported in part by a PGRP grant from the National Science Foundation (DBI $\# 0077617$ ). Work in the Dong lab is supported in part by the Waksman Institute of Microbiology and Rutgers University, and by an NIH grant (R01GM109080).

\section{REFERENCES}

Aida, M., Beis, D., Heidstra, R., Willemsen, V., Blilou, I., Galinha, C., et al. (2004). The PLETHORA genes mediate patterning of the Arabidopsis root stem cell niche. Cell 119, 109-120. doi: 10.1016/j.cell.2004.09.018

Apostolou, E., Ferrari, F., Walsh, R. M., Bar-Nur, O., Stadtfeld, M., Cheloufi, S., et al. (2013). Genome-wide chromatin interactions of the Nanog locus in pluripotency, differentiation, and reprogramming. Cell Stem Cell 12, 699-712. doi: 10.1016/j.stem.2013.04.013

Beisel, C., and Paro, R. (2011). Silencing chromatin: comparing modes and mechanisms. Nat. Rev. Genet. 12, 123-135. doi: 10.1038/nrg2932

Belmont, A. S. (2014). Large-scale chromatin organization: the good, the surprising, and the still perplexing. Curr. Opin. Cell Biol. 26, 69-78. doi: 10.1016/j.ceb.2013.10.002

Bergmann, J. H., and Spector, D. L. (2014). Long non-coding RNAs: modulators of nuclear structure and function. Curr. Opin. Cell Biol. 26, 10-18. doi: 10.1016/j.ceb.2013.08.005

Berman, B. P., Weisenberger, D. J., Aman, J. F., Hinoue, T., Ramjan, Z., Liu, Y., et al. (2011). Regions of focal DNA hypermethylation and long-range hypomethylation in colorectal cancer coincide with nuclear lamina-associated domains. Nat. Genet. 44, 40-46. doi: 10.1038/ng.969

Burton, J. N., Adey, A., Patwardhan, R. P., Qiu, R., Kitzman, J. O., and Shendure, J. (2013). Chromosome-scale scaffolding of de novo genome assemblies based on chromatin interactions. Nat. Biotechnol. 31, 1119-1125. doi: 10.1038/nbt.2727

Chen, T., and Dent, S. Y. (2014). Chromatin modifiers and remodellers: regulators of cellular differentiation. Nat. Rev. Genet. 15, 93-106. doi: 10.1038/nrg3607

Chuang, C. H., Carpenter, A. E., Fuchsova, B., Johnson, T., de Lanerolle, P., and Belmont, A. S. (2006). Long-range directional movement of an interphase chromosome site. Curr. Biol. 16, 825-831. doi: 10.1016/j.cub.2006.03.059

Cowan, C. R., Carlton, P. M., and Cande, W. Z. (2001). The polar arrangement of telomeres in interphase and meiosis. Rabl organization and the bouquet. Plant Physiol. 125, 532-538. doi: 10.1104/pp.125.2.532

Crevillén, P., Sonmez, C., Wu, Z., and Dean, C. (2013). A gene loop containing the floral repressor FLC is disrupted in the early phase of vernalization. EMBO J. 32, 140-148. doi: 10.1038/emboj.2012.324

Creyghton, M. P., Cheng, A. W., Welstead, G. G., Kooistra, T., Carey, B. W., Steine, E. J., et al. (2010). Histone H3K27ac separates active from poised enhancers and predicts developmental state. Proc. Natl. Acad. Sci. U.S.A. 107, 21931-21936. doi: 10.1073/pnas.1016071107

Deal, R. B., and Henikoff, S. (2010). A simple method for gene expression and chromatin profiling of individual cell types within a tissue. Dev. Cell 18, 10301040. doi: 10.1016/j.devcel.2010.05.013

Dekker, J., Rippe, K., Dekker, M., and Kleckner, N. (2002). Capturing chromosome conformation. Science 295, 1306-1311. doi: 10.1126/science.1067799

Deng, W., Lee, J., Wang, H., Miller, J., Reik, A., Gregory, P. D., et al. (2012). Controlling long-range genomic interactions at a native locus by targeted tethering of a looping factor. Cell 149, 1233-1244. doi: 10.1016/j.cell.2012.03.051

Denholtz, M., Bonora, G., Chronis, C., Splinter, E., de Laat, W., Ernst, J., et al. (2013). Long-range chromatin contacts in embryonic stem cells reveal a role for pluripotency factors and polycomb proteins in genome organization. Cell Stem Cell 13, 602-616. doi: 10.1016/j.stem.2013.08.013

de Wit, E., Bouwman, B. A., Zhu, Y., Klous, P., Splinter, E., Verstegen, M. J., et al. (2013). The pluripotent genome in three dimensions is shaped around pluripotency factors. Nature 501, 227-231. doi: 10.1038/nature12420

Dixon, J. R., Selvaraj, S., Yue, F., Kim, A., Li, Y., Shen, Y., et al. (2012). Topological domains in mammalian genomes identified by analysis of chromatin interactions. Nature 485, 376-380. doi: 10.1038/nature11082

Dong, F., and Jiang, J. (1998). Non-Rabl patterns of centromere and telomere distribution in the interphase nuclei of plant cells. Chromosome Res. 6, 551-559. doi: 10.1023/A:1009280425125 
Galinha, C., Hofhuis, H., Luijten, M., Willemsen, V., Blilou, I., Heidstra, R., et al. (2007). PLETHORA proteins as dose-dependent master regulators of Arabidopsis root development. Nature 449, 1053-1057. doi: 10.1038/nature06206

Gallois, J. L., Woodward, C., Reddy, G. V., and Sablowski, R. (2002). Combined SHOOT MERISTEMLESS and WUSCHEL trigger ectopic organogenesis in Arabidopsis. Development 129, 3207-3217. doi: 10.1038/nature06206

Ginno, P., and Schubeler, D. (2013). Twisting chromatin in stem cells. EMBO J. 32, 2304-2306. doi: 10.1038/emboj.2013.179

Grob, S., Schmid, M. W., Luedtke, N. W., Wicker, T., and Grossniklaus, U. (2013). Characterization of chromosomal architecture in Arabidopsis by chromosome conformation capture. Genome Biol. 14, R129. doi: 10.1186/gb-2013-14$11-\mathrm{r} 129$

Haseloff, J. (1999). GFP variants for multispectral imaging of living cells. Methods Cell Biol. 58, 139-151. doi: 10.1016/S0091-679X(08)61953-6

Heun, P., Laroche, T., Shimada, K., Furrer, P., and Gasser, S. M. (2001). Chromosome dynamics in the yeast interphase nucleus. Science 294, 2181-2186. doi: 10.1126/science. 1065366

Jin, F., Li, Y., Dixon, J. R., Selvaraj, S., Ye, Z., Lee, A. Y., et al. (2013). A high-resolution map of the three-dimensional chromatin interactome in human cells. Nature 503 290-294. doi: 10.1038/nature12644

Jovtchev, G., Borisova, B. E., Kuhlmann, M., Fuchs, J., Watanabe, K., Schubert, I., et al. (2011). Pairing of lacO tandem repeats in Arabidopsis thaliana nuclei requires the presence of hypermethylated, large arrays at two chromosomal positions, but does not depend on H3-lysine-9-dimethylation. Chromosoma 120, 609-619. doi 10.1007/s00412-011-0335-8

Jovtchev, G., Watanabe, K., Pecinka, A., Rosin, F. M., Mette, M. F., Lam, E., et al. (2008). Size and number of tandem repeat arrays can determine somatic homologous pairing of transgene loci mediated by epigenetic modifications in Arabidopsis thaliana nuclei. Chromosoma 117, 267-276. doi: 10.1007/s00412-0070146-0

Kagey, M. H., Newman, J. J., Bilodeau, S., Zhan, Y., Orlando, D. A., van Berkum, N. L., et al. (2010). Mediator and cohesin connect gene expression and chromatin architecture. Nature 467, 430-435. doi: 10.1038/nature09380

Kaplan, N., and Dekker, J. (2013). High-throughput genome scaffolding from in vivo DNA interaction frequency. Nat. Biotechnol. 31, 1143-1147. doi $10.1038 /$ nbt. 2768

Kato, N., and Lam, E. (2001). Detection of chromosomes tagged with green fluorescent protein in live Arabidopsis thaliana plants. Genome Biol. 2, RESEARCH0045 doi: 10.1186/gb-2001-2-11-research0045

Katz, A., Oliva, M., Mosquna, A., Hakim, O., and Ohad, N. (2004). FIE and CURLY LEAF polycomb proteins interact in the regulation of homeobox gene expression during sporophyte development. Plant J. 37, 707-719. doi: 10.1111/j.1365-313X.2003.01996.x

Kieffer-Kwon, K. R., Tang, Z., Mathe, E., Qian, J., Sung, M. H., Li, G., et al. (2013). Interactome maps of mouse gene regulatory domains reveal basic principles of transcriptional regulation. Cell 155, 1507-1520. doi: 10.1016/j.cell.2013.11.039

Kornet, N., and Scheres, B. (2008). Stem cell factors in plants: chromatin connections. Cold. Spring Harb. Symp. Quant. Biol. 73, 235-242. doi $10.1101 / \mathrm{sqb} .2008 .73 .043$

Krijger, P. H., and de Laat, W. (2013). Identical cells with different 3D genomes; cause and consequences? Curr. Op. Genet. Dev. 23, 191-196. doi: 10.1016/j.gde.2012.12.010

Kwon, C. S., Chen, C., and Wagner, D. (2005). WUSCHEL is a primary target for transcriptional regulation by SPLAYED in dynamic control of stem cell fate in Arabidopsis. Genes Dev. 19, 992-1003. doi: 10.1101/gad.1276305

Lam, E., Kato, N., and Watanabe, K. (2004). Visualizing chromosome structure/organization. Annu. Rev. Plant Biol. 55, 537-544. doi: 10.1146/annurev.arplant.55.031903.141807

Lee, E., Lucas, J. R., Goodrich, J., and Sack, F. D. (2014). Arabidopsis guard cell integrity involves the epigenetic stabilization of the FLP and FAMA transcription factor genes. Plant J. 78, 566-577. doi: 10.1111/tpj.12516

Lessard, J. A., and Crabtree, G. R. (2010). Chromatin regulatory mechanisms in pluripotency. Annu. Rev. Cell Dev. Biol. 26, 503-532. doi: 10.1146/annurevcellbio-051809-102012

Li, G., Ruan, X., Auerbach, R. K., Sandhu, K. S., Zheng, M., Wang, P., et al. (2012). Extensive promoter-centered chromatin interactions provide a topological basis for transcription regulation. Cell 148, 84-98. doi: 10.1016/j.cell.2011. 12.014
Lieberman-Aiden, E., van Berkum, N. L., Williams, L., Imakaev, M., Ragoczy, T., Telling, A., etal. (2009). Comprehensive mapping of long-range interactions reveals folding principles of the human genome. Science 326, 289-293. doi: $10.1126 /$ science.1181369

Louwers, M., Bader, R., Haring, M., van Driel, R., de Laat, W., and Stam, M. (2009). Tissue- and expression level-specific chromatin looping at maize b1 epialleles. Plant Cell 21, 832-842. doi: 10.1105/tpc.108.064329

Mattout, A., and Meshorer, E. (2010). Chromatin plasticity and genome organization in pluripotent embryonic stem cells. Curr. Opin. Cell Biol. 22, 334-341. doi: 10.1016/j.ceb.2010.02.001

Meissner, A. (2010). Epigenetic modifications in pluripotent and differentiated cells. Nat. Biotechnol. 28, 1079-1088. doi: 10.1038/nbt.1684

Meshorer, E., Yellajoshula, D., George, E., Scambler, P. J., Brown, D. T., and Misteli, T. (2006). Hyperdynamic plasticity of chromatin proteins in pluripotent embryonic stem cells. Dev. Cell 10, 105-116. doi: 10.1016/j.devcel.2005.10.017

Misteli, T. (2007). Beyond the sequence: cellular organization of genome function. Cell 128, 787-800. doi: 10.1016/j.cell.2007.01.028

Nora, E. P., Lajoie, B. R., Schulz, E. G., Giorgetti, L., Okamoto, I., Servant, N., et al. (2012). Spatial partitioning of the regulatory landscape of the X-inactivation centre. Nature 485, 381-385. doi: 10.1038/nature11049

Papp, B., and Plath, K. (2013). Epigenetics of reprogramming to induced pluripotency. Cell 152, 1324-1343. doi: 10.1016/j.cell.2013.02.043

Phillips-Cremins, J. E., Sauria, M. E., Sanyal, A., Gerasimova, T. I., Lajoie, B. R., Bell, J. S., etal. (2013). Architectural protein subclasses shape 3D organization of genomes during lineage commitment. Cell 153, 1281-1295. doi: 10.1016/j.cell.2013.04.053

Rosa, S., De Lucia, F., Mylne, J. S., Zhu, D., Ohmido, N., Pendle, A., et al. (2013). Physical clustering of FLC alleles during Polycomb-mediated epigenetic silencing in vernalization. Genes Dev. 27, 1845-1850. doi: 10.1101/gad.221713.113

Rosin, F. M., Watanabe, N., Cacas, J. L., Kato, N., Arroyo, J. M., Fang, Y., et al. (2008). Genome-wide transposon tagging reveals location-dependent effects on transcription and chromatin organization in Arabidopsis. Plant J. 55, 514-525. doi: 10.1111/j.1365-313X.2008.03517.x

Sabatini, S., Heidstra, R., Wildwater, M., and Scheres, B. (2003). SCARECROW is involved in positioning the stem cell niche in the Arabidopsis root meristem. Genes Dev. 17, 354-358. doi: 10.1101/gad.252503

Sablowski, R. (2004). Plant and animal stem cells: conceptually similar, molecularly distinct? Trends Cell Biol. 14, 605-611. doi: 10.1016/j.tcb.2004.09.011

Sang, Y., Wu, M. F., and Wagner, D. (2009). The stem cell - chromatin connection. Semin. Cell Dev. Biol. 20, 1143-1148. doi: 10.1016/j.semcdb.2009.09.006

Santos, A. P., and Shaw, P. (2004). Interphase chromosomes and the Rabl configuration: does genome size matter? J. Microsc. 214, 201-206. doi: 10.1111/j.0022-2720.2004.01324.x

Schubert, D., Primavesi, L., Bishopp, A., Roberts, G., Doonan, J., Jenuwein, T., et al. (2006). Silencing by plant Polycomb-group genes requires dispersed trimethylation of histone $\mathrm{H} 3$ at lysine 27. EMBO J. 25, 4638-4649. doi: 10.1038/sj.emboj.7601311

Selvaraj, S., Dixon, J. R., Bansal, V., and Ren, B. (2013). Whole-genome haplotype reconstruction using proximity-ligation and shotgun sequencing. Nat. Biotechnol. 31, 1111-1118. doi: 10.1038/nbt.2728

Sexton, T., Yaffe, E., Kenigsberg, E., Bantignies, F., Leblanc, B., Hoichman, M., et al. (2012). Three-dimensional folding and functional organization principles of the Drosophila genome. Cell 148, 458-472. doi: 10.1016/j.cell.2012. 01.010

Shen, W. H., and Xu, L. (2009). Chromatin remodeling in stem cell maintenance in Arabidopsis thaliana. Mol. Plant 2, 600-609. doi: 10.1093/mp/ ssp022

Singh, M. B., and Bhalla, P. L. (2006). Plant stem cells carve their own niche. Trends Plant Sci. 11, 241-246. doi: 10.1016/j.tplants.2006.03.004

Steiner, F. A., Talbert, P. B., Kasinathan, S., Deal, R. B., and Henikoff, S. (2012). Cell-type-specific nuclei purification from whole animals for genome-wide expression and chromatin profiling. Genome Res. 22, 766-777. doi: 10.1101/gr. 131748.111

Thiagarajan, R. D., Morey, R., and Laurent, L. C. (2014). The epigenome in pluripotency and differentiation. Epigenomics 6, 121-137. doi: 10.2217/epi.13.80 Vastenhouw, N. L., Zhang, Y., Woods, I. G., Imam, F., Regev, A., Liu, X. S., et al. (2010). Chromatin signature of embryonic pluripotency is established during genome activation. Nature 464, 922-926. doi: 10.1038/nature08866 
Visel, A., Blow, M. J., Li, Z., Zhang, T., Akiyama, J. A., Holt, A., et al. (2009). ChIP-seq accurately predicts tissue-specific activity of enhancers. Nature 457, 854-858. doi: 10.1038 /nature 07730

Voigt, P., Tee, W. W., and Reinberg, D. (2013). A double take on bivalent promoters. Genes Dev. 27, 1318-1338. doi: 10.1101/gad.219626.113

Watanabe, K., Pecinka, A., Meister, A., Schubert, I., and Lam, E. (2005). DNA hypomethylation reduces homologous pairing of inserted tandem repeat arrays in somatic nuclei of Arabidopsis thaliana. Plant J. 44, 531-540. doi: 10.1111/j.1365313X.2005.02546.x

Wei, Z., Gao, F., Kim, S., Yang, H., Lyu, J., An, W., et al. (2013). Klf4 organizes long-range chromosomal interactions with the oct4 locus in reprogramming and pluripotency. Cell Stem Cell 13, 36-47. doi: 10.1016/j.stem.2013. 05.010

Wright, J. E., and Ciosk, R. (2013). RNA-based regulation of pluripotency. Trends Genet. 29, 99-107. doi: 10.1016/j.tig.2012.10.007

Young, R. A. (2011). Control of the embryonic stem cell state. Cell 144, 940-954. doi: 10.1016/j.cell.2011.01.032

Zhang, J., Poh, H. M., Peh, S. Q., Sia, Y. Y., Li, G., Mulawadi, F. H., et al. (2012). ChIAPET analysis of transcriptional chromatin interactions. Methods 58, 289-299. doi: 10.1016/j.ymeth.2012.08.009
Zhang, Y., Wong, C. H., Birnbaum, R. Y., Li, G., Favaro, R., Ngan, C. Y., et al. (2013). Chromatin connectivity maps reveal dynamic promoter-enhancer longrange associations. Nature 504, 306-310. doi: 10.1038/nature12716

Conflict of Interest Statement: The authors declare that the research was conducted in the absence of any commercial or financial relationships that could be construed as a potential conflict of interest.

Received: 31 May 2014; accepted: 11 July 2014; published online: 21 August 2014.

Citation: Luo C, Dong J, Zhang Y and Lam E (2014) Decoding the role of chromatin architecture in development: coming closer to the end of the tunnel. Front. Plant Sci. 5:374. doi: 10.3389/fpls.2014.00374

This article was submitted to Plant Genetics and Genomics, a section of the journal Frontiers in Plant Science.

Copyright (c) 2014 Luo, Dong, Zhang and Lam. This is an open-access article distributed under the terms of the Creative Commons Attribution License (CC BY). The use, distribution or reproduction in other forums is permitted, provided the original author(s) or licensor are credited and that the original publication in this journal is cited, in accordance with accepted academic practice. No use, distribution or reproduction is permitted which does not comply with these terms. 